\title{
Co/Cu/Co Pseudo Spin-Valve System Prepared by Magnetron Sputtering with Different Argon Pressure
}

\author{
A.V. Svalovi,2,a , A.N. Sorokin ${ }^{2}$, P.A. Savin ${ }^{2, b}$, A. García-Arribas ${ }^{1, c}$, \\ A. Fernández ${ }^{3}$, V.O. Vas'kovskiy ${ }^{2}$ and G.V. Kurlyandskaya ${ }^{1}$ \\ ${ }^{1}$ Departamento de Electricidad y Electrónica, Universidad del País Vasco (UPV/EHU), 48080 \\ Bilbao, Spain \\ ${ }^{2}$ Department of Magnetism and Magnetic Nanomaterials, Ural Federal University, 620002 \\ Ekaterinburg, Russia \\ ${ }^{3}$ Departamento de Física, Universidad de Oviedo, 33007 Oviedo, Asturias, Spain \\ aandrey.svalov@ehu.es, bpeter.savin@urfu.ru, calf@we.lc.ehu.es
}

Keywords: Coercive force, Magnetoresistance, Magnetron sputtering, Co films and multilayers, Spin-valve.

\begin{abstract}
Thin Co films were fabricated by DC magnetron sputtering. The effect of argon pressure on the microstructure, surface morphology and magnetic properties of the samples was systematically studied. It was found that with the increase of argon pressure, the sharpness of the crystalline texture of the samples declines, the roughness of film surfaces and the coercivity of the films increase. Based on these results, a $\mathrm{Co} / \mathrm{Cu} / \mathrm{Co}$ pseudo spin-valve system was designed and the corresponding structures were fabricated. The difference in coercivity of magnetic layers was obtained by deposition of the Co layers at different Ar pressures. Change of the resistance of this trilayer is induced at a moderate field by the spin rotation in the soft layer with lower coercivity.
\end{abstract}

\section{Introduction}

In past years, spin-valve devices have been employed for various applications: measurements of electrical current, high-density magnetic recording heads, position sensing applications, non-volatile magnetic random access memories. Spin-valve systems (in their simplest form) consist of three magnetic layers [1]. The first one is a magnetically soft ferromagnetic layer, the magnetization of which can be easily rotated by the external magnetic field. The second ferromagnetic layer is separated from the first by a spacer of non-magnetic metal. The magnetization of the second layer should remain unaffected by the external field. It is pinned by the exchange bias interaction with the third magnetic (antiferromagnetic or ferrimagnetic) layer. The electrical resistance of a spin-valve increases with the change of the angle between the magnetizations of the reciprocal magnetic layers from a parallel to an antiparallel alignment (giant magnetoresistance (GMR)) [2]. However, GMR can be observed in simpler systems which consist of two magnetic layers separated by a nonmagnetic spacer - so-called pseudo spin-valve system [3-5]. The difference between the coercive fields $\left(H_{\mathrm{c}}\right)$ of the two layers allows a rotation of the magnetization of one layer. At the same time, the magnetization of another layer can be kept unchanged under the application of a certain external field. The difference in the coercive fields can be achieved by using the layers of various magnetic materials with different $H_{\mathrm{c}}$ value, for example, FeNi and Co [5]. Moreover, the same material can be used for both magnetic layers, but in this case the layers must be deposited under different preparation conditions [2]. It is well known, that magnetic properties of thin films depend on film structure, which in turn is determined by the deposition parameters: substrate temperature, deposition rate, argon pressure [6,7]. In this way coercive force of thin films can be changed over a wide range.

In this paper, we study the effect of argon pressure on structure and magnetic properties of Co thin films and a $\mathrm{Co} / \mathrm{Cu} / \mathrm{Co}$ pseudo spin-valve system prepared on their basis. 


\section{Experimental details}

The multilayred samples were deposited by magnetron sputtering onto glass substrates at room temperature. Background pressure was $3 \times 10^{-7}$ mbar. The argon pressures $\left(p_{\text {Ar }}\right)$ during deposition were selected from the available interval of $1.9 \times 10^{-3}$ to $2.4 \times 10^{-2}$ mbar. A constant magnetic field of 200 Oe was applied during thin film deposition parallel to the film plane in order to induce a uniaxial magnetic anisotropy. The microstructure was studied by X-ray diffraction (XRD) using PHILIPS X'PERT PRO automatic diffractometer operating at $40 \mathrm{kV}$ and $40 \mathrm{~mA}$, in theta-theta configuration, secondary monochromator with $\mathrm{Cu}-\mathrm{K} \alpha$ radiation. Atomic force microscopy was used to determine the topographical characteristics of the films. The hysteresis loops were recorded by means of the magneto-optic Kerr effect (MOKE). The magnetoresistance was measured following the conventional four-point probe technique with the in-plane magnetic field oriented along the easy magnetization axis.

\section{Results and discussion}

It was observed that the deposition rate $\left(V_{\text {dep }}\right)$ of the Co films varied with the working gas pressure (Fig. 1). It means that the number of sputtered particles that arrive at the substrate decreases at higher $p_{\text {Ar }}$, reducing the deposition rate. Moreover, the oblique angle of the deposited particles increases due to the scattering of the sputtered atoms by the working gas atoms, the average kinetic energy of the deposited atoms decreases [8]. Therefore it can be expected that $\mathrm{Ar}$ pressure change will affect the structure of the films. Fig. 2 shows the $\theta-2 \theta$ XRD spectra for $80-\mathrm{nm}-$ thick Co films deposited at three different working pressures $\left(1.9 \times 10^{-3}, 3.7 \times 10^{-3}\right.$ and $2.4 \times 10^{-2}$ mbar). For the samples deposited at relatively low argon pressure the Miller indexes of the peaks can be identified with the reflections of the hcp-Co phase. With reducing pressure, the (002) peak intensity was gradually increased and a (002) texture was developed. The average grain size calculated by Scherrer method [9] is approximately $30 \mathrm{~nm}$ for the films deposited at the lowest pressure. With the argon pressure increase, the average grain size decreases. At highest pressure, the XRD pattern of the film is almost featureless, suggesting that it is nano-crystalline or amorphouslike state. At high pressure, the reduced energy of arrived particles leads to reduced surface mobility of the deposited atoms and a higher nucleation density. Therefore, the grain size decreases with the increase of the argon pressure [5].
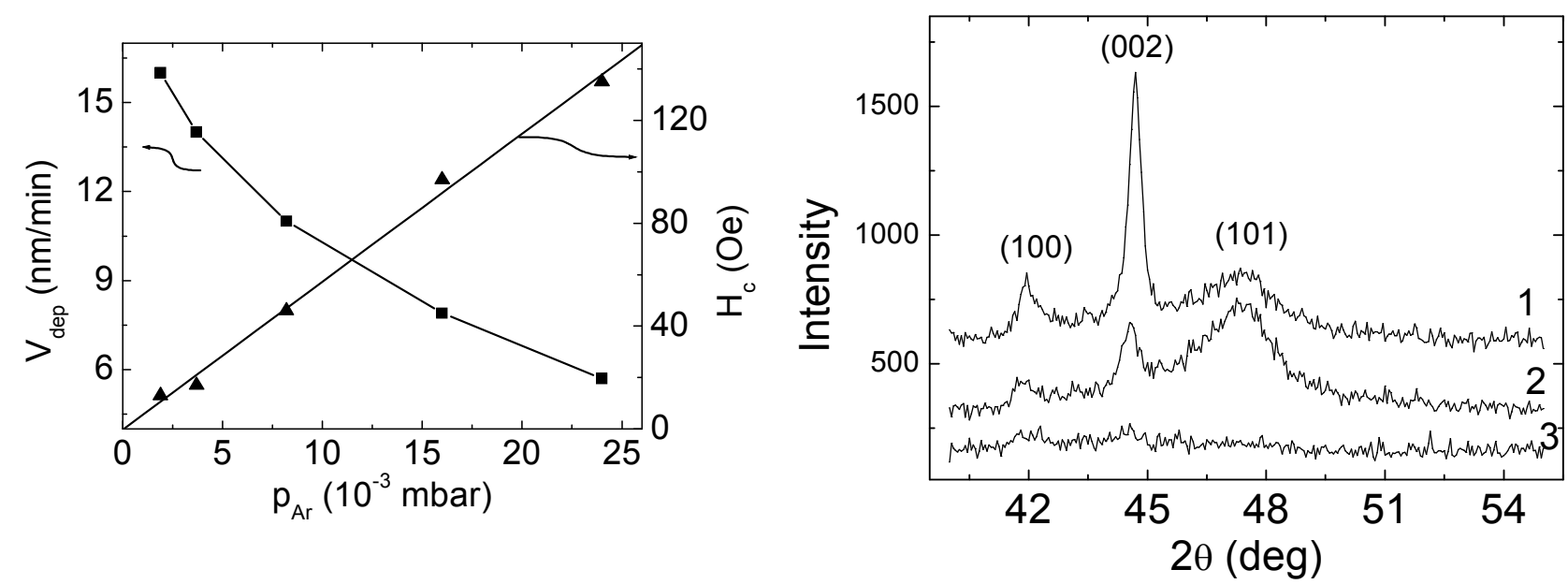

Fig. 1. Ar pressure dependence of the deposition rate and the coercive force of Co films which was measured for films with a thickness of $10 \mathrm{~nm}$.

Fig. 2. X-ray diffraction spectra for $\operatorname{Co}(80 \mathrm{~nm})$ films deposited at different Ar pressure: $1.9 \times 10^{-3} \operatorname{mbar}(1), 3.7 \times 10^{-3} \operatorname{mbar}(2)$, $2.4 \times 10^{-2}$ mbar (3). 
a

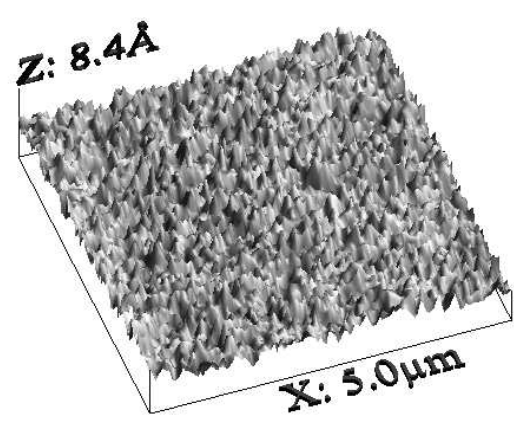

$\mathrm{R}_{\mathrm{rms}}=0.1 \mathrm{~nm}$ b

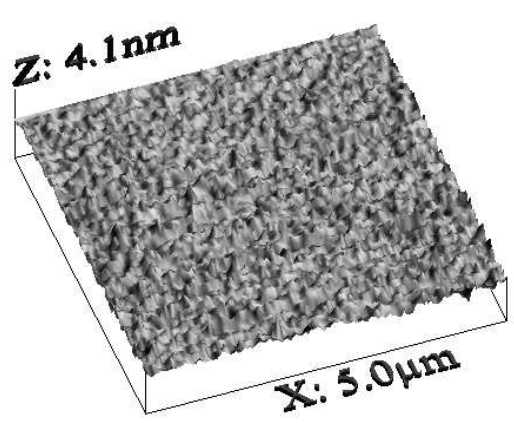

$\mathrm{R}_{\mathrm{rms}}=0.3 \mathrm{~nm}$
$\mathrm{C}$

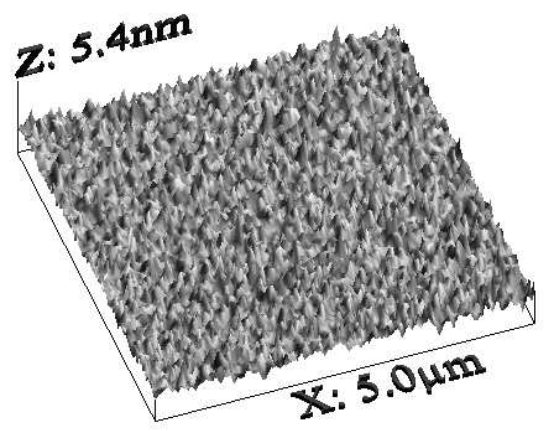

$\mathrm{R}_{\mathrm{rms}}=0.6 \mathrm{~nm}$

Fig. 3. AFM images for $10 \mathrm{~nm}$ Co films deposited at different Ar pressure: $1.9 \times 10^{-3} \mathrm{mbar}(\mathrm{a})$, $3.7 \times 10^{-3} \mathrm{mbar}(\mathrm{b}), 2.4 \times 10^{-2} \mathrm{mbar}(\mathrm{c})$. The values of rms roughness $\left(R_{\mathrm{rms}}\right)$ are indicated, too.

Broadening the oblique angle of the deposited particles of the angular distribution leads to intergrain shading, which gives rise to a morphology with clusters and voids [5]. The AFM images indicate that the topography of the sample surface changes appreciably and the root-mean-square roughness $\left(R_{\mathrm{rms}}\right)$ increases with argon pressure increase (Fig. 3). If particles arrive to the substrate with higher oblique angle, the surface becomes rougher because the voids will have a limited angle of acceptance for the deposited particles and the deposition will occur preferentially on the higher peaks [8].

It is logical to expect that the features of both bulk structure and surface morphology will affect the coercive force of the films. Fig. 1 shows the dependence of coercivity $\left(H_{\mathrm{c}}\right)$ versus $p_{\text {Ar }}$ for $10 \mathrm{~nm}$ thick Co films. It is clearly seen that $H_{\mathrm{c}}$ increases with decreasing $p_{\mathrm{Ar}}$. This $H_{\mathrm{c}}$ dependence on the pressure is suggested to be related not only to structural features, but also to the change of inner stress of the films [10].

Keeping in mind the $H_{\mathrm{c}}$ dependence on $p_{\text {Ar }}$, very simple pseudo spin-valve system can be obtained by DC magnetron sputtering using only the $\mathrm{Co}$ and $\mathrm{Cu}$ targets. In our case the nominal sample composition was $\mathrm{Co}(10 \mathrm{~nm}) / \mathrm{Cu}(3 \mathrm{~nm}) / \mathrm{Co}(10 \mathrm{~nm})$. The choice of the $\mathrm{Cu}$ layer thickness was based on two criteria. On the one hand, in order to enhance the MR ratio it is necessary to reduce the $\mathrm{Cu}$ interlayer thickness. On the other hand, the $\mathrm{Cu}$ interlayer thickness should be sufficient to guarantee the suppression of the interlayer coupling. On the basis of previous results discussed in the literature, $3 \mathrm{~nm} \mathrm{Cu}$ interlayer spacer was used [11]. Fig. 4 shows the MOKE hysteresis loop for such a trilayer. Here, the bottom Co layer with higher coercivity deposited at highest $p_{\mathrm{Ar}}$ is a magnetically hard layer and the top Co layer deposited at lowest $p_{\text {Ar }}$ is a soft layer.

During magnetization reversal of the whole sandwich, as the first step, the magnetization of the top Co layer reverses and therefore there is a small field regime where the alignment of the magnetizations of two magnetic layers is antiparallel. Fig. 5 shows the associated field dependence of the resistance $R(H)$. The resistance changes as a function of the external magnetic field are induced by spin-dependent scattering and their increase is due to the antiparallel magnetizations' alignment.

\section{Summary}

Structural characteristics and magnetic properties of thin Co films prepared by magnetron sputtering were studied. It was found that the effect of the Ar pressure on the coercivity of the Co films is related mainly to the microstructures and surface morphology of these films. On the basis of the results, the $\mathrm{Co} / \mathrm{Cu} / \mathrm{Co}$ trilayers were designed and prepared. An antiparallel alignment of 


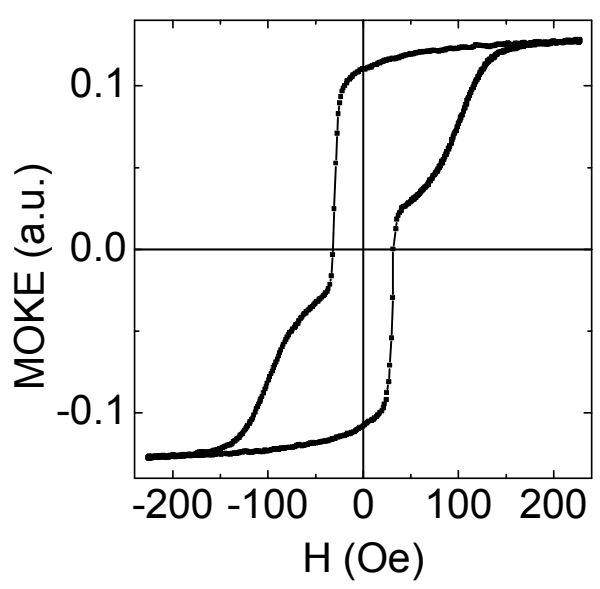

Fig. 4. MOKE hysteresis loop for the $\mathrm{Co} / \mathrm{Cu} / \mathrm{Co}$ trilayer.

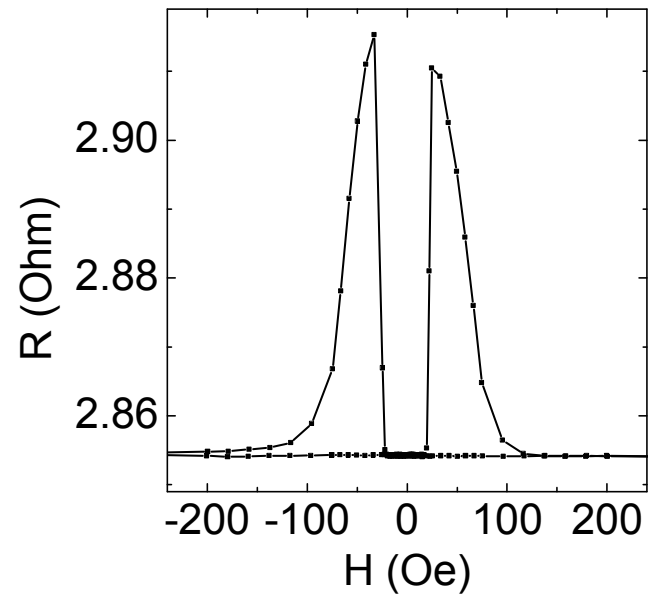

Fig. 5. Magnetoresistance curve for the $\mathrm{Co} / \mathrm{Cu} / \mathrm{Co}$ trilayer.

magnetizations in two Co layers can be formed because the coercivities of these layers are different due to the deposition at different Ar pressures. Thereby, conditions for realization of the GMR effect are insured. Thus, it was demonstrated that argon pressure is an effective tool for preparation of $\mathrm{Co} / \mathrm{Cu} / \mathrm{Co}$ pseudo spin-valves.

\section{Acknowledgment}

This work was supported by the Spanish Government grant MAT2011-27573-C04, Russian Ministry of Education and Science, (contract No 02.G36.31.0004), and ACTIMAT project of Etortek programme of the Basque Government and UPV-EHU. Selected measurements were done at SGIKER services of UPV-EHU.

\section{References}

[1] M. Johnson (Editor): Magnetoelectronics (Academic Press, The Netherlands 2004).

[2] P. Grünberg and R. Coehoorn, in: Magnetic Multilayers and Giant Magnetoresistance. Fundamentals and Industrial Applications, edited by U. Hartman (Springer-Verlag, Germany 2000).

[3] M. Jergel, Y. Halahovets, P. Šiffalovič, K. Végsö, R. Senderák, E. Majková, Š. Luby: Thin Solid Films Vol. 520 (2011), p. 667.

[4] A.V. Svalov, A. Fernández, M. Tejedor, G.V. Kurlyandskaya: Vacuum Vol. 81 (2007), p. 1012.

[5] A. Paul, T. Damm, D.E. Bürgler, S. Stein, H. Kohlstedt, and P. Grünberg: Appl. Phys. Lett. Vol. 82 (2003), p. 1905.

[6] J.A. Thornton : J. Vac. Sci. Technol. Vol. 11 (1974), p. 666.

[7] A.V. Svalov, I.R. Aseguinolaza, A. García-Arribas, I. Orue, J.M. Barandiaran, J. Alonso, M.L. Fdez-Gubieda, G.V. Kurlyandskaya: IEEE Trans. Magn. Vol. 46 (2010), p. 333.

[8] V.P. Nascimento, E.C. Passamani, A.D. Alvarenga, A. Biondo, F. Pelegrini, E. Baggio Saitovitch: Appl. Surf. Sci. 254 (2008), p. 2114.

[9] A.L. Patterson: Phys. Rev. Vol. 56 (1939), p. 978.

[10] F. Xu, Q. Huang, Z. Liao, S. Li, and C. K. Ong: J. Appl. Phys. 111 (2012), 07A304.

[11] A.V. Svalov, P.A. Savin, G.V. Kurlyandskaya, J. Gutiérrez, J.M. Barandiarán and V.O. Vas'kovskiy: IEEE Trans. Magn. Vol. 38 (2002), p. 2782. 